\title{
Electrical properties of individual tin oxide nanowires contacted to platinum electrodes
}

\author{
F. Hernandez-Ramirez, ${ }^{1}$ A. Tarancon, ${ }^{1}$ O. Casals, ${ }^{1}$ E. Pellicer, ${ }^{1}$ J. Rodriguez,${ }^{1}$ A. Romano-Rodriguez, ${ }^{1}$ J. R. Morante, ${ }^{1}$ \\ S. Barth, 2,3 and S. Mathur $2,3, *$ \\ ${ }^{1} I N^{2} U B$ and EME/CERMAE/CEMIC Departament d'Electronica, Universitat de Barcelona UB, \\ C/Marti I Franqués 1, E-08028 Barcelona, Spain \\ ${ }^{2}$ Department of Inorganic Chemistry, Wuerzburg University, 97074 Wuerzburg, Germany \\ ${ }^{3}$ Department of Nanocrystalline Materials and Thin Film Systems, Leibniz Institut für Neue Materialien, \\ D-66123 Saarbruecken, Germany \\ (Received 26 February 2007; published 22 August 2007)
}

\begin{abstract}
A simple and useful experimental alternative to field-effect transistors for measuring electrical properties (free electron concentration $n_{d}$, electrical mobility $\mu$, and conductivity $\sigma$ ) in individual nanowires has been developed. A combined model involving thermionic emission and tunneling through interface states is proposed to describe the electrical conduction through the platinum-nanowire contacts, fabricated by focused ion beam techniques. Current-voltage $(I-V)$ plots of single nanowires measured in both two- and four-probe configurations revealed high contact resistances and rectifying characteristics. The observed electrical behavior was modeled using an equivalent circuit constituted by a resistance placed between two back-to-back Schottky barriers, arising from the metal-semiconductor-metal $(M-S-M)$ junctions. Temperature-dependent $I-V$ measurements revealed effective Schottky barrier heights up to $\Phi_{B E}=0.4 \mathrm{eV}$.
\end{abstract}

DOI: 10.1103/PhysRevB.76.085429 PACS number(s): 74.78. - w, 73.63.Bd, 73.61.Le, 73.40.Sx

\section{INTRODUCTION}

Fabrication of nanodevices with enhanced performance still poses a key challenge for the realization of new nanometer-scale electronics and devices, which, in turn, depends on the better understanding of the electrical properties of interfaces between metal nanoelectrodes and onedimensional (1D) nanomaterials. ${ }^{1}$ Nanowires, nanotubes, and nanobelts exhibit unique physical, optical, and electrical properties due to diminishing dimensions and associated confinement effects,${ }^{1-3}$ however, the integration of these tiny objects in functional devices necessitates better understanding of their intrinsic properties. ${ }^{4}$ In the context of 1D nanostructures, formation of electrical nanocontacts with high stability, low contact resistance $\left(R_{C}\right)$, and Ohmic behavior are among the critical issues. Focused ion beam (FIB)-assisted lithography techniques have been demonstrated to be useful for manifold applications, including fabrication of electrical contacts, without an external mask, in nanodevices. ${ }^{5-7}$ Estimation of electrical parameters of individual 1D nanowires (NWs) using FIB-assisted nanolithography has been demonstrated in recent reports. ${ }^{8-10}$ Nevertheless, the formation of Schottky barriers at the metal-NW interfaces, when semiconductor materials are contacted, complicates the nanodevice characterization due to non-Ohmic electrical responses and high contact resistances. ${ }^{11,12}$

We have investigated an effective alternative to using conventional field-effect transistor (FET) geometries, which have been used to determine key parameters of $\mathrm{SnO}_{2} \mathrm{NWs}$ in the past. ${ }^{4,13}$ In this work, individual tin oxide NWs were electrically contacted using FIB nanolithography techniques to study and model the electrical response in individual nanowires. Tin oxide, an $n$-type semiconductor due to intrinsic oxygen vacancies, was chosen due to its low-cost synthesis and large number of potential application areas. ${ }^{14}$ The main conduction mechanism in the nanocontacts was shown to be the combination of thermionic emission and tunneling phenomenon, assisted by interface states, through the metal-NW junctions. Stability problems associated with the existence of Schottky barriers at the metal-semiconductor $(M-S)$ junctions are also discussed.

\section{EXPERIMENTAL DETAILS}

$\mathrm{SnO}_{2} \mathrm{NWs}$ with radial dimensions around $20 \mathrm{~nm}$ were synthesized by chemical vapor deposition of a molecular precursor $\left[\mathrm{Sn}\left(\mathrm{O}^{t} \mathrm{Bu}\right)_{4}\right]$ following a method reported elsewhere. ${ }^{15}$ Transmission electron microscopy investigations revealed the main growth direction to be [100] with interplanar spacing corresponding to the rutile structure of $\mathrm{SnO}_{2}$ and displaying high quality single crystalline wire bodies. ${ }^{15}$ Some of these NWs were dispersed over the surface of $\mathrm{SiO}_{2} / \mathrm{Si}$ substrates with photolithographically prepatterned $\mathrm{Au} / \mathrm{Ti} / \mathrm{Ni}$ microelectrodes. The as-dispersed NWs were electrically contacted using a FEI Strata 235 dual beam instrument equipped with a trimethyl(methylcyclopentadienyl)-platinum(IV) $\left[\mathrm{C}_{5} \mathrm{H}_{4} \mathrm{CH}_{3} \mathrm{Pt}\left(\mathrm{CH}_{3}\right)_{3}\right]$ injector to deposit $\mathrm{Pt}$ (Fig. 1). The electron and $\mathrm{Ga}^{+}$beams were accelerated to 5 and $30 \mathrm{kV}$, respectively. The procedure for this contact fabrication method is explained in detail elsewhere. ${ }^{10}$ Two- and four-probe dc electrical measurements were performed using a Keithley 2400 source measure unit. All measurements were carried out in a ProboStat cell placed inside a furnace to control the working temperature, which was monitored by a thermocouple located next to the sample. ${ }^{16}$ The experimental setup (cell and furnace) was found to act as a Faraday cup which avoids electrical measurements from being affected by external electromagnetic noise. We tested in this study 30 individual devices, which showed consistent results apart from a small number $(<10 \%)$ in which the performance was influenced by slight material modification, contact instabilities (FIB preparation), or twinning in NWs. 


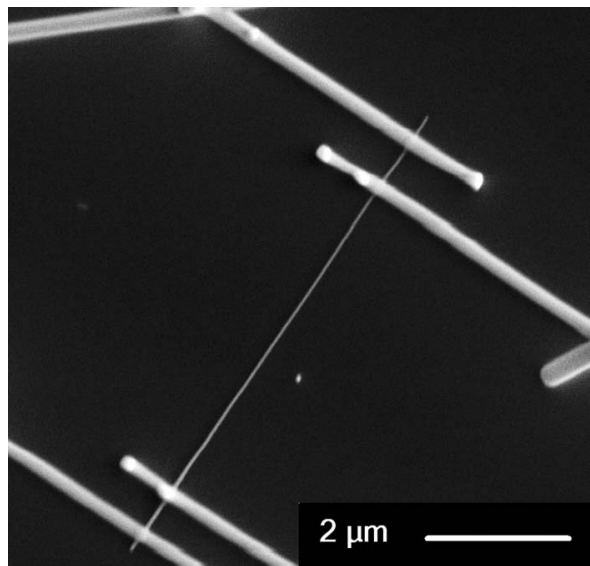

FIG. 1. $\mathrm{SnO}_{2}$ nanowire of radius $r=27 \pm 3 \mathrm{~nm}$ and length $L$ $=11 \mu \mathrm{m}$ electrically contacted using dual-beam FIB nanolithography techniques.

\section{RESULTS AND DISCUSSION}

\section{A. Two-probe $I-V-T$ measurements}

Two-probe measurements performed on $\mathrm{SnO}_{2} \mathrm{NWs}$ at room temperature (RT) produced slightly rectifying currentvoltage $(I-V)$ characteristics (Fig. 2), which can be explained considering the existence of two back-to-back Schottky barriers connected in series to both extremes of the nanowires. ${ }^{17}$ Conduction through these devices is dominated by reversebiased junctions and, therefore, it is the major contribution to the total contact resistance (Fig. 3). On the other hand, forward-biased junctions offer good electrical conduction and low contact resistance. For two-probe dc measurements, the device resistance $R$ can be described as the sum of the resistance arising from the reverse-biased junction $R_{S I}$, the nanowire resistance $R_{N W}$, the forward-biased junction resistance $R_{S D}$, and resistance contribution of the rest of the elements of the circuit, $R_{\mathrm{Pt}}$, such as Pt stripes or cables (Fig. 3). If $R_{S D}$ and $R_{\mathrm{Pt}}$ are small enough compared to $R_{S I}$ and $R_{N W}$ the device resistance $R$ can be simplified to

$$
R=R_{S I}+R_{N W}
$$

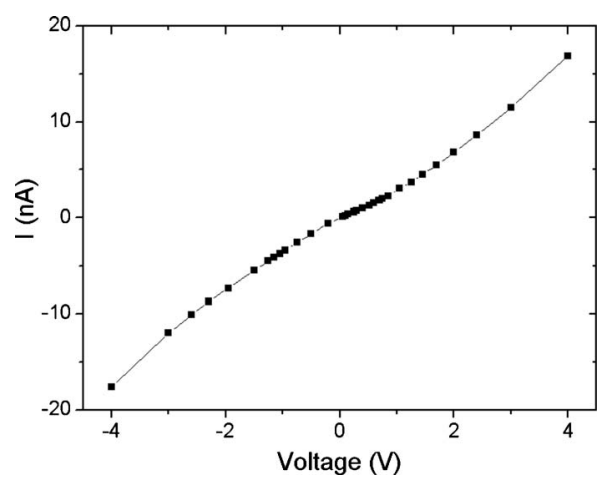

FIG. 2. $I-V$ curve of an individual NW (Fig. 1) at $T=21{ }^{\circ} \mathrm{C}$ exhibiting a slightly rectifying response.

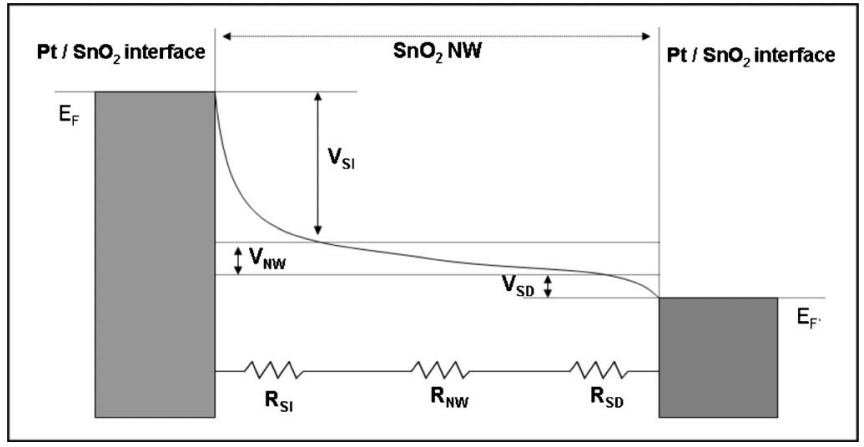

FIG. 3. Band diagram of the back-to-back Schottky configurations existing in FIB contacted NWs. Voltage drops are indicated and related to the resistances used in the equivalent circuit.

Although our devices present non-Ohmic characteristics, resistance $R$ can be locally defined and calculated as follows:

$$
R=\left(\frac{\partial I}{\partial V}\right)_{V_{0}}^{-1},
$$

where $I$ is the measured current, $V$ the total applied voltage, and $V_{0}$ the voltage at a fixed point. The diminishing device resistance with increasing $V$ can be clearly observed in Fig. 4.

Voltage drop at reverse-biased junction $V_{S I}$ can be estimated using the following expression:

$$
V_{S I}=V\left(1-\frac{R_{N W}}{R}\right)=V\left(1-\frac{R_{N W}}{R_{S I}+R_{N W}}\right) .
$$

According to Eq. (3), an accurate modeling of reverse-biased junctions deduced from their $I-V_{S I}$ characteristics requires precise estimation of nanowire resistance $\left(R_{N W}\right)$ values. In the past, some reports have assumed that nanowire resistances $R_{N W}$ are negligible against resistance associated with reverse-biased junctions $R_{S I}$, mistaking $V_{S I}$ for the total applied voltage $V$, which can lead to erroneous conclusions, especially if $R_{N W}$ is of the same order of magnitude as $R_{S I}$. Therefore, four-probe measurements are mandatory in esti-

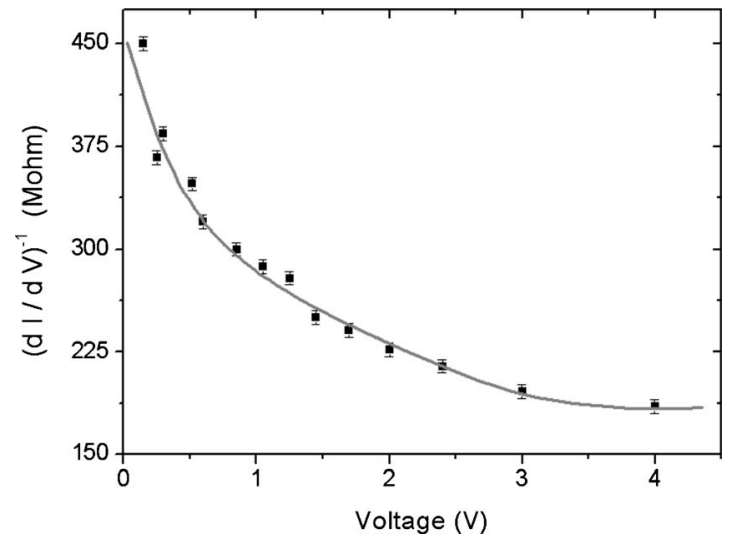

FIG. 4. Two-probe resistance of the NW (Fig. 1) as a function of the applied voltage $V$ at $T=21{ }^{\circ} \mathrm{C}$. Reduction of $R$ occurs when electrons overcome the reverse-biased junction more easily due to a decreased barrier height caused by increasing voltage. 
TABLE I. Resistance $R_{N W}$ and conductivity $\sigma$ of the NW of Fig. 1, obtained in four-probe measurements at different temperatures $T$.

\begin{tabular}{lcc}
\hline \hline $\begin{array}{l}\text { Temperature } \\
(\mathrm{K})\end{array}$ & $\begin{array}{c}R_{N W} \\
\left(10^{6} \Omega\right)\end{array}$ & $\begin{array}{c}\sigma \\
(\Omega \mathrm{cm})^{-1}\end{array}$ \\
\hline 294 & 76 & 0.46 \\
333 & 65 & 0.54 \\
393 & 34 & 1.03 \\
453 & 14 & 2.50 \\
513 & 2.7 & 12.95 \\
\hline \hline
\end{tabular}

mating $R_{N W}$, since the contribution of Schottky resistance $R_{S I}$ can be eliminated.

The observed rectifying behavior at room temperature $\left(T=21^{\circ} \mathrm{C}\right.$; Fig. 2) tends to disappear above $T=180{ }^{\circ} \mathrm{C}$, and perfect Ohmic curves were consistently observed at $T$ $=240{ }^{\circ} \mathrm{C}$, suggesting that at this temperature, conduction electrons possess enough mobility to overcome the energy barrier existing in reverse-biased junctions.

\section{B. Four-probe measurements}

Four-probe current-voltage $(I-V)$ measurements were performed on $\mathrm{SnO}_{2} \mathrm{NWs}$ under synthetic air in the temperature range $21-240{ }^{\circ} \mathrm{C}$. Semiconductor properties of $\mathrm{SnO}_{2} \mathrm{NWs}$ were confirmed by the observed negative temperature coefficient behavior, whereby resistance $R_{N W}$ was found to decrease with increasing $T$ (Table I).

The estimated electrical conductivity $\sigma$ of our $\mathrm{SnO}_{2} \mathrm{NWs}$ ranged from 0.1 to $0.9(\Omega \mathrm{cm})^{-1}$ at $T=21{ }^{\circ} \mathrm{C}$ depending on the device and was found to increase by 1 order of magnitude at $T=240{ }^{\circ} \mathrm{C}$ (Table I and Fig. 5). The observed dispersion in $\sigma$ suggested that the electrical properties of $\mathrm{SnO}_{2}$ NWs depend on the mode of synthesis, compositional purity, and surface chemistry. The observed conductivity values were in accordance to previous results published for $\mathrm{SnO}_{2}$ NWs contacted by electron-beam nanolithography

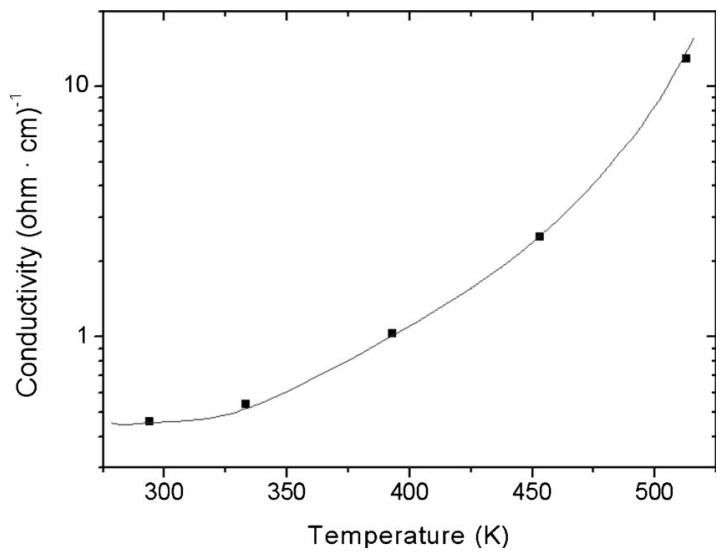

FIG. 5. Electrical conductivity $\sigma$ of the NW shown in Fig. 1 as a function of increasing temperature $T$. The observed negative temperature coefficient is due to the semiconducting properties of $\mathrm{SnO}_{2}$.
TABLE II. Experimental data used to model the reverse-biased junction characteristics at $T=21{ }^{\circ} \mathrm{C}$ of the NW shown in Fig. 1. The first two columns $(V, I)$ represent the total applied voltage in twoprobe experiments and obtained current. $R_{S I}$ is the resistance of reverse-biased junction $\left(R_{S I}=R-R_{N W}=R-76 \mathrm{M} \Omega\right)$ and, finally, the voltage drop in the junction $V_{S I}$, calculated by Eq. (4).

\begin{tabular}{lccc}
\hline \hline $\begin{array}{l}V \\
(\mathrm{~V})\end{array}$ & $\begin{array}{c}I \\
\left(10^{-9} \mathrm{~A}\right)\end{array}$ & $\begin{array}{c}R_{S I} \\
\left(10^{6} \Omega\right)\end{array}$ & $\begin{array}{c}V_{S I} \\
(\mathrm{~V})\end{array}$ \\
\hline 1 & 3.50 & 211 & 0.735 \\
1.5 & 6.00 & 174 & 1.044 \\
2 & 8.90 & 149 & 1.324 \\
2.5 & 12.02 & 132 & 1.587 \\
3 & 15.08 & 123 & 1.854 \\
3.5 & 18.05 & 118 & 2.129 \\
4 & 21.62 & 109 & 2.357 \\
\hline \hline
\end{tabular}

techniques. ${ }^{18}$ The estimation of $R_{N W}$ can be employed to determine the $I-V_{S I}$ characteristics [Eq. (3)] of reverse-biased junctions, which allows a correct modeling of the conduction mechanisms (Table II).

\section{Modeling of Schottky barriers and determination of nanowire parameters}

The current flow in a Schottky barrier junction is caused by the transport of charge carriers from semiconductor to metal or in the reverse direction. The carrier transport can occur by different mechanisms, such as (a) thermionic emission over the barrier, (b) tunneling assisted by interface states existing in the $M-S$ junctions, and (c) pure tunneling through the barrier. ${ }^{19}$

$I-V_{S I}$ characteristics at room temperature of real $\mathrm{Pt}_{-} \mathrm{SnO}_{2}$ junctions can be usually modeled with the help of thermionic emission and tunneling assisted by interface states. According to this combined model, the contact barrier height is reduced and the current increases with increasing bias according to the following expression: ${ }^{20,21}$

$$
I=A A^{* *} T^{2} \exp \left(\frac{-q \phi_{B E}}{k_{B} T}\right),
$$

where

$$
\phi_{B E}=\phi_{B 0}-\sqrt{\frac{q E}{4 \pi \varepsilon_{s}}}-\frac{q}{\varepsilon_{S}} \sqrt{\frac{N_{S} d}{4 \pi}}
$$

and

$$
E=\sqrt{\frac{2 q n_{d}}{\varepsilon_{s}}\left(V_{S I}+\phi_{b i}-\frac{k_{B} T}{q}\right)},
$$

where $A$ is the contact area, $A^{* *}$ is the effective Richardson constant, $\phi_{B E}$ is the effective barrier height, $\phi_{B 0}$ is the ideal barrier height in the absence of image force, $E$ is the maximum electric field at the junction, $\varepsilon_{s}$ and $n_{d}$ are the dielectric constant and free electron concentration of $\mathrm{SnO}_{2}, \phi_{b i}$ is the built-in potential, $N_{S}$ is the interface state concentration, and $d$ is the introduced depth of these defects. When the concen- 


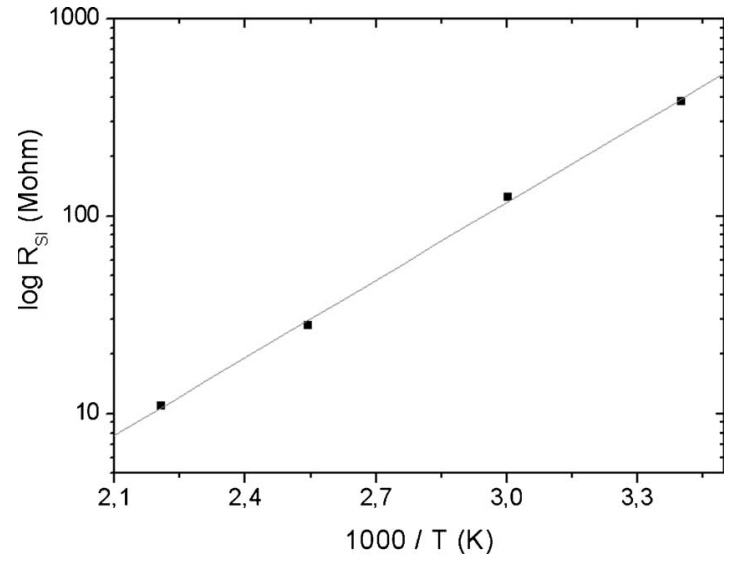

FIG. 6. $\log (I)$ vs $\left(V_{S I}\right)$ plot of the NW shown in Fig. 1 at $T$ $=21{ }^{\circ} \mathrm{C}$. The linear behavior demonstrated that the experimental data fit with the proposed conduction model (line). NW free carrier concentration $\left(n_{d} \sim 10^{18} \mathrm{~cm}^{-3}\right)$ was calculated from the observed slope.

tration of the interface state increases the tunneling current becomes more significant and superimposes on the thermionic emission current, resulting in an apparent lowering of the Schottky barrier height. This being the case, a plot of $\ln (I)$ vs $\left(V_{S I}\right)^{1 / 4}$ should be linear at all temperatures for which the proposed conduction mechanism here is correct. Figure 6 shows the validity of this model for our experimental data.

The Schottky effective barrier height $\phi_{B E}$ of our $M-S$ contacts was estimated by plotting the evolution of reversebiased junction resistances $R_{S I}$ at a fixed voltage $V_{S I}$, as a function of temperature $T$. Both magnitudes are related according to the following activation-energy law:

$$
R_{S I} \propto \exp \left(\frac{q \phi_{B E}}{k_{B} T}\right) .
$$

According to Eq. (7), $\phi_{B E}$ can be obtained from the slope of the $\ln \left(R_{S I}\right)$ vs $1 / T$ plot (Fig. 6). In our experiments, effective barrier height $\left(\phi_{B E}\right)$ was found to be below $0.4 \mathrm{eV}$, which is significantly lower than the expected theoretical value $\left(\Phi_{B 0}\right.$ $=0.75 \pm 0.10 \mathrm{eV}$ ) for clean Pt-SnO 2 interfaces,${ }^{22}$ suggesting that tunneling assisted by interface states is not a negligible term in the conduction of reverse-biased junctions. The necessity of accounting for both tunneling and thermionic contributions can be attributed to defects induced by electron bombardment and metal organic precursor decomposition in the contact regions. The existence of some of these defects can justify the observed Ohmic responses at room temperature in some of our devices.

A reliable estimation of free electron concentration $\left(n_{d}\right)$ in $\mathrm{SnO}_{2} \mathrm{NWs}$ is a nontrivial process. FET experiments have been traditionally used as the most common solution to determine $n_{d}$, a NW key parameter required for the development of nanodevices with improved characteristics. ${ }^{4,13}$ Using Eqs. (3)-(5), it was demonstrated that $n_{d}$ can be obtained from the slope of the $\ln (I)$ vs $\left(V_{S I}\right)^{1 / 4}$ plot at a fixed temperature $T$ (Fig. 6). Our devices show average values for free carrier concentrations $n_{d} \sim 10^{18} \mathrm{~cm}^{-3}$ at room temperature and values for electron mobility $\mu$ between 1 and $10 \mathrm{~cm}^{2} / \mathrm{V} \mathrm{s}$ calculated using the following relationship:

$$
\sigma=q n_{d} \mu .
$$

Considering the approximation that electron mobility $\mu$ is a constant parameter in the measured temperature range $\left(21-240{ }^{\circ} \mathrm{C}\right)$ and for a given free carrier concentration, the observed increase in conductivity $(\sigma)$ as a function of temperature $T$ can be attributed to rise of free carrier concentration $n_{d}$ in $\mathrm{SnO}_{2}$ NWs. According to this assumption, $n_{d}$ values of $\sim 10^{20} \mathrm{~cm}^{-3}$ were obtained in our NWs at $240{ }^{\circ} \mathrm{C}$, which is highly conform with the values reported in literature, ${ }^{13}$ demonstrating the suitability of this approach for estimating semiconductor characteristics of NWs.

The results obtained in this study demonstrate the possibility of using Schottky barrier measurements to characterize the electrical properties of NWs, however, the presented model does not consider several factors, such as image force effects in Eq. (6) and self-heating effects in NWs, which should be accounted for, in order to obtain more precise results. Dissipated power $P$ in such nanosystems can increase their effective temperature $T$ by Joule heating effect that can be calculated by

$$
P=I V=\frac{V^{2}}{R}=I^{2} R .
$$

If the wire cannot dissipate the power through conduction and radiation, the wire will decay in the reverse-biased contact region where the highest resistances occur in our devices. The calculated power density at the contact region is 2 orders of magnitude higher, when compared to that at the rest of the wire body. However, an estimation of the effective contact area is merely a rough approximation, due to the presence of high carbon content in the Pt contacts. Therefore, Joule heating causes an intrinsic error in the estimation of key parameters such as $n_{d}$ or $\mu$ in most of the works related to NWs. This problem is even more important if the studied nanosystems have rectifying contacts where a great part of the applied voltage drops at $M-S$ contacts. Furthermore, par-

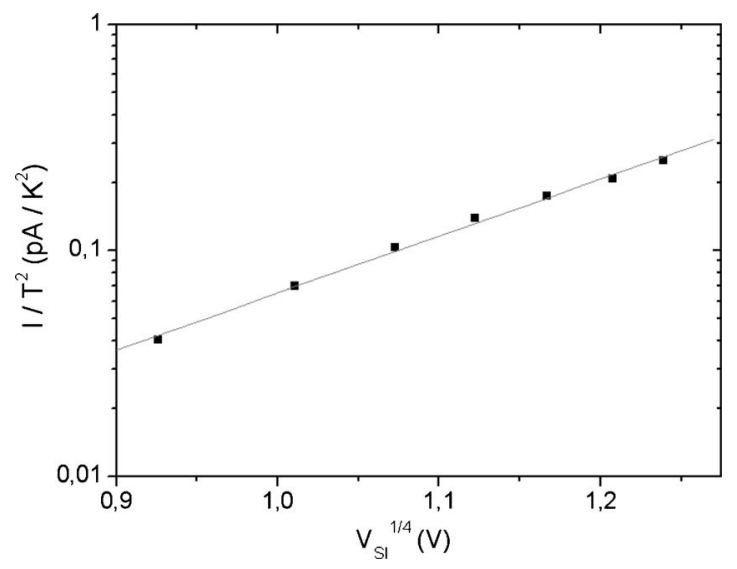

FIG. 7. Reverse-biased Schottky resistance $R_{S I}$ of NW (Fig. 1) as a function of the temperature. Based on the experimental data, the effective Schottky barrier height was found to be $\Phi_{B E}$ $=0.28 \pm 0.02 \mathrm{eV}$. 


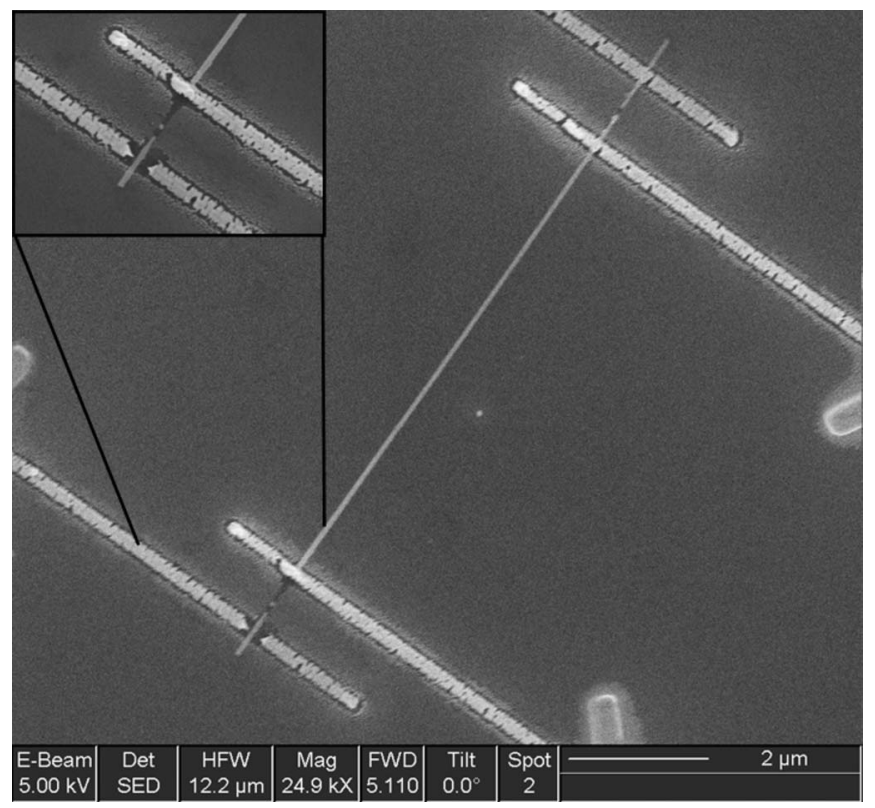

FIG. 8. SEM image of $\mathrm{SnO}_{2} \mathrm{NW}$ (shown in Fig. 1) after a few hours under working conditions. The piece of NW placed between the inner contacts is not affected by self-heating effects; however, the contact area (inset) is destroyed. Melting temperature of $\mathrm{SnO}_{2}$ is close to $T=1100{ }^{\circ} \mathrm{C}$, demonstrating that local temperature close to the Schottky barriers can be much higher.

tial degradation of platinum contacts can occur due to evaporation of the carbon content existing in FIB-produced platinum stripes, which is manifested in the appearance of small holes and cracks. ${ }^{23}$ Consequently, a catastrophic failure of nanodevices is sometimes observed after prolonged operation periods, which appears as disintegration of contacts and nanowires (Figs. 7 and 8).

\section{CONCLUSION}

Individual tin oxide NWs have been contacted using FIB nanolithography techniques to fabricate NW-based electrical circuits. Two-probe current-voltage $(I-V)$ measurements revealed slightly rectifying characteristics which tend to disappear with increasing temperature. Four-probe measurements enabled us to estimate NW conductivity in the temperature range $21-240{ }^{\circ} \mathrm{C}$. Rectifying behavior is explained by considering the existence of two back-to-back Schottky barriers in the Pt- $\mathrm{SnO}_{2}$ junctions, modeled considering both thermionic emission and charge carrier tunneling assisted by interface states. The proposed model allowed accurate estimation of free electron concentration $\left(n_{d}\right)$ or electron mobility $(\mu)$. The obtained parameters were consistent with results reported using FET experiments, demonstrating that our approach is a viable alternative to these experiments. Finally, the influence of self-heating process in contacted NWs is presented, which can cause destruction of operating nanodevices especially at the nanocontact areas.

\section{ACKNOWLEDGMENTS}

This work has been partially supported by the EU through the project NANOS4 of the VI FMP, the Human Potential Program Access to Research Infrastructures, and the projects MAGASENS and CROMINA. F.H.-R and O.C. are indebted to the Spanish Ministry of Education (MEC) for the FPU grant. S.M. and S.B. thank the Saarland State for providing the necessary infrastructure facilities. Thanks are due to the German Science Foundation (DFG) for supporting this work in the frame of the priority program on nanomaterials, Sonderforschungbereich 277 at the Saarland University, Saarbruecken, Germany.
*FAX: 49-931-888-4619; s.mathur@uni-wuerzburg.de

${ }^{1}$ J. Jortner and C. N. R. Rao, Pure Appl. Chem. 74, 1489 (2002).

${ }^{2}$ G. Schmidt, Nanoparticles: From Theory to Application (Wiley, New York, 2004).

${ }^{3}$ M. Law, J. Goldberger, and P. Yang, Annu. Rev. Mater. Res. 34, 83 (2004).

${ }^{4}$ A. Kolmakov and M. Moskovits, Annu. Rev. Mater. Res. 34, 151 (2004).

${ }^{5}$ K. Gamo, Semicond. Sci. Technol. 8, 1118 (1993).

${ }^{6}$ S. Reyntjens and R. Puers, J. Micromech. Microeng. 11, 287 (2001).

${ }^{7}$ G. de Marzi, D. Iacopino, A. J. Quinn, and G. Redmond, J. Appl. Phys. 96, 3458 (2004).

${ }^{8}$ T. Choi, D. Poulikakos, J. Tharian, and U. Sennhauser, Appl. Phys. Lett. 87, 013108 (2005).

${ }^{9}$ S. Valizadeh, M. Abid, F. Hernandez-Ramirez, A. RomanoRodriguez, K. Hjort, and J. A. Schweitz, Nanotechnology 17, 1134 (2006).

${ }^{10}$ F. Hernandez-Ramirez, J. Rodriguez, O. Casals, E. Russinyol, A. Vila, A. Romano-Rodriguez, J. R. Morante, and M. Abid, Sens. Actuators B 118, 198 (2006).

${ }^{11}$ C. Y. Nam, D. Tham, and J. E. Fischer, Nano Lett. 5, 2029
(2005).

${ }^{12}$ Z. Y. Zhang, C. H. Jin, X. L. Liang, Q. Chen, and L. P. Peng, Appl. Phys. Lett. 88, 073102 (2006).

${ }^{13}$ Y. Zhang, A. Kolmakov, Y. Lilach, and M. Moskovits, J. Phys. Chem. B 109, 1923 (2006).

${ }^{14}$ N. Barsan and U. Weimar, J. Phys.: Condens. Matter 15, R813 (2003).

${ }^{15}$ S. Mathur, S. Barth, H. Shen, J.-C. Pyun, and U. Werner, Small 1, 713 (2005).

${ }^{16} \mathrm{http}: / /$ www.norecs.com/

${ }^{17}$ F. Hernandez-Ramirez et al., Nanotechnology 17, 5577 (2006).

${ }^{18}$ M. S. Arnold, P. Avouris, Z. W. Pan, and Z. L. Wang, J. Phys. Chem. B 107, 659 (2006).

${ }^{19}$ B. L. Sharma, Metal Semiconductors Schottky Barrier Junctions and their Applications (Plenum, New York, 1984).

${ }^{20}$ S. M. Sze, Physics of Semiconductor Devices (Wiley, New York, 1981).

${ }^{21}$ J. M. Shannon, Appl. Phys. Lett. 24, 8 (1973).

${ }^{22}$ H. Elhouichet, A. Moadhen, M. Oueslati, S. Romhdane, J. R. Roger, and H. Bouchriha, Phys. Status Solidi C 9, 3349 (2005).

${ }^{23}$ A. Botman, J. J. L. Mulders, R. Weemaes, and S. Mentink, Nanotechnology 17, 3779 (2006). 